\title{
An analytic model for the epoch of halo creation
}

\author{
W.J.Percival, ${ }^{1,2}$ L.Miller $^{1}$ and J.A.Peacock ${ }^{2}$ \\ 1 Dept. of Physics, University of Oxford, Nuclear $\&$ Astrophysics Laboratory, Keble Road, Oxford OX1 3RH, U.K. \\ ${ }^{2}$ Institute for Astronomy, University of Edinburgh, Royal Observatory, Blackford Hill, Edinburgh EH9 3HJ, U.K.
}

Submitted for publication in MNRAS

\begin{abstract}
In this paper we describe the Bayesian link between the cosmological mass function and the distribution of times at which isolated halos of a given mass exist. By assuming that clumps of dark matter undergo monotonic growth on the time-scales of interest, this distribution of times is also the distribution of 'creation' times of the halos. This monotonic growth is an inevitable aspect of gravitational instability. The spherical top-hat collapse model is used to estimate the rate at which clumps of dark matter collapse. This gives the prior for the creation time given no information about halo mass. Applying Bayes' theorem then allows any mass function to be converted into a distribution of times at which halos of a given mass are created. This general result covers both Gaussian and non-Gaussian models. We also demonstrate how the mass function and the creation time distribution can be combined to give a joint density function, and discuss the relation between the time distribution of major merger events and the formula calculated. Finally, we determine the creation time of halos within three N-body simulations, and compare the link between the mass function and creation rate with the analytic theory.
\end{abstract}

Key words: galaxies: halos - formation, cosmology: theory - dark matter

\section{INTRODUCTION}

The hierarchical build-up of self-gravitating dark matter is though to drive evolution in the observable universe. The formation of clumps of dark matter precipitates the formation of galaxies by providing a potential well into which gas can fall and subsequently cool. Violent mergers between equally sized halos and their associated galaxies are thought to be important for starbursts and quasar activation. In order to model and understand the observable universe it is therefore essential to understand the build-up of the dark structure.

The most widely used analytic model for the distribution of mass in isolated halos at any epoch comes from PressSchechter (PS) theory (Press \& Schechter 1974). By smoothing the initial field of density fluctuations on different scales, information on the distribution of perturbation sizes can be obtained. Linking the time at which these perturbations collapse to the initial overdensities using the simplified spherical top-hat collapse model allows the distribution of mass in isolated halos at any epoch to be determined (Press \& Schechter 1974; Peacock \& Heavens 1990; Bond et al. 1991).

In Percival \& Miller (1999) (hereafter paper I), we used the tenets of PS theory to model the related, but distinct problem of determining the distribution of times at which halos of a given mass are created. Here, 'creation' is defined as the epoch at which non-linear collapse is predicted. Two derivations were given, one of which directly used the trajectories invoked in PS theory (Peacock \& Heavens 1990; Bond et al. 1991), and one of which used Bayes' theorem to convert from the PS mass function to a time distribution. The second derivation required the prior for the creation time which was calculated by examining the trajectories model.

In this paper we extend the Bayesian link between the mass function and the creation time distribution to cover any mass function. This is important, not only because it is known that standard PS theory is wrong in detail (e.g. Sheth \& Tormen 1999), but especially because the new extension applies to mass functions derived from more general density fields including non-Gaussian models (e.g. Matarrese, Verde \& Jimenez 2000).

First, we adopt the assumption that all clumps monotonically increase in mass on the cosmological time scales of interest. This monotonic growth is an inevitable aspect of gravitational instability. Every epoch should now be thought of as a creation time for a given clump, and we need not make the distinction between the creation time distribution and the distribution of times at which a given halo exists.

In order to convert from a mass function to a distribution in time we require the prior for the creation time. This is the rate at which creation events occur, given no information about the halo mass. In this work we use the spherical 
top-hat collapse (STHC) model to provide a simple mechanism for determining the required rate. In Section 2 we derive the link between collapse time and the overdensity at an early epoch for the STHC model within any Friedmann cosmology. Having determined that this relation is independent of halo mass, this leads directly to an approximation to the prior for the creation time, described in Section 3. This is the second major assumption adopted in this paper: that the prior for the creation time is well approximated by this simple model for the break-away of structure from linear expansion. This means that following the two simple assumptions detailed above, we are able to convert any mass function to give the distribution of epochs at which halos of a given mass are created.

Simple models of cosmologically evolving phenomena often adopt an important mass range rather than a specific halo mass (e.g. paper I, Granato et al. 1999). In order to use the work presented here in these models, the joint distribution of halos in mass and creation time is required. Although calculating the required joint probability is formally impossible because the equations cannot be properly normalised, a formula with the correct shape can be determined and is presented in Section 5 .

So far we have not made a distinction between the slow accretion of mass onto a halo and major mergers between halos. Such a distinction is important because only major mergers are thought to play a vital role in starbursts and quasar activation (see paper I). The time distribution calculated in this paper determines when halos existed (or were created by any mechanism assuming monotonic clump growth) which is not necessarily equal to the distribution of merger events. This is discussed in Section 6 .

Finally, we compare the analytic link between the mass function and the creation rate to the results from three numerical simulations of structure formation in different cosmological models. An analytic fit to the mass function as described by Sheth \& Tormen (1999) is adopted and is converted into a creation rate using the STHC model. This model is compared with and shown to be in good agreement with the numerical results.

\section{THE SPHERICAL TOP-HAT COLLAPSE MODEL}

In this Section we analyse the STHC model which is the simplest model for the way in which clumps of dark matter break free from linear growth and undergo non-linear collapse. We present the derivation of the link between the initial overdensity and the collapse time $t_{\text {coll }}$ in a form which clearly shows that this link is independent of the mass of the overdensity. The derivation also demonstrates a method for calculating this link within any Friedmann cosmology. Similar derivations have been previously discussed in a variety of subsets of this space: for an Einstein-de Sitter model, a derivation is given by Gunn \& Gott (1972), for an open $\Omega_{V}=0$ model by Lacey \& Cole (1993), and for a flat $\Omega_{V} \neq 0$ universe by Eke Cole \& Frenk (1996). A summary of these results is given in Kitayama \& Suto (1996). A numerical prescription for the calculation of the overdensity in any cosmology has also been developed (Somerville \& Primack 1999).
These derivations all use the same basic idea which is adopted in this work: the behaviour of two spheres of equal mass is compared within the cosmological framework. One of the spheres evolves with the background density $\rho_{b}(t)$, while the other is perturbed by a uniform excess density $\Delta \rho(t)$. In subsequent analysis, a subscript ' $b$ ' denotes that a quantity relates to the sphere with background density, and ' $p$ ' to the perturbation.

Matter is assumed to be an ideal fluid with no pressure and the universe is modelled as spherically symmetric around the perturbation. As a consequence of Birkhoff's theorem, the gravitational field of both the perturbation and the background is described by a Robertson-Walker (RW) metric with curvature constant $K$, and RW scale factor $a(t)$. The behaviour of such perturbations is governed by Friedmann's equation which we will consider in the form:

$$
\left(\frac{d a}{d t}\right)^{2}+K=\frac{2 G M}{a}+\left(H_{0}^{2} \Omega_{V}\right) a^{2}
$$

where $M$ is the mass inside the sphere. Note that in order to compare spheres with different behaviour, we do not normalise the scale factor $a(t)$ to equal the curvature scale (by dividing by $\sqrt{|K|}$ ) so $K$ is allowed to take any real value.

To calculate the behaviour of the overdensity at an early time, we note that a series solution for $a(t)$ in the limit $t \rightarrow 0$ can be obtained for Equation 11. This is given by $a=\alpha t^{2 / 3}+\beta t^{4 / 3}+O\left(t^{6 / 3}\right)$, where:

$\alpha=\left(\frac{9 G M}{2}\right)^{1 / 3}, \quad \beta=\frac{3 K}{20}\left(\frac{6}{G M}\right)^{1 / 3}$.

Using the fact that the spheres contain equal mass, the behaviour of $\delta(t) \equiv \Delta \rho(t) / \rho_{b}(t)$ in the limit $t \rightarrow 0$ is given by:

$$
\begin{aligned}
\lim _{t \rightarrow 0}\left(\frac{\Delta \rho(t)}{\rho_{b}(t)}\right) & =\lim _{t \rightarrow 0}\left(\frac{a_{b}(t)^{3}}{a_{p}(t)^{3}}-1\right) \\
& =\frac{3}{\alpha}\left(\beta_{p}-\beta_{b}\right) t^{2 / 3}+O\left(t^{4 / 3}\right) .
\end{aligned}
$$

Defining:

$\epsilon=\frac{K}{\left(G M H_{0}\right)^{2 / 3}}$,

the present day normalisation of Equation 1 gives that for a sphere of uniform background density:

$\epsilon_{b}=\left(\Omega_{M}+\Omega_{V}-1\right)\left(\frac{2}{\Omega_{M}}\right)^{2 / 3}$,

We can now combine Equations 2, 3 \& 5 to determine the behaviour of $\delta(t)$ in the limit $t \rightarrow 0$ as a function of $\epsilon_{p}$ :

$$
\begin{aligned}
\lim _{t \rightarrow 0} \delta(t) & =\frac{9}{20}\left(\frac{4}{3}\right)^{1 / 3} \\
& \times\left[\left(\Omega_{M}+\Omega_{V}-1\right)\left(\frac{2}{\Omega_{M}}\right)^{2 / 3}-\epsilon_{p}\right]\left(H_{0} t\right)^{2 / 3} \\
& +O\left[\left(H_{0} t\right)^{4 / 3}\right]
\end{aligned}
$$

If the field of perturbations is linearly extrapolated to present day and normalised here, the approximation of Carroll, Press \& Turner 1992 for the ratio of the current linear amplitude to the Einstein-de Sitter model can be used to extrapolate the limiting behaviour of $\delta$ to this epoch. The extrapolated limit, $\delta_{\lim }$, is related to $\epsilon_{p}$ by: 


$$
\begin{aligned}
\delta_{\lim } & \simeq \frac{3}{8}\left(4 \Omega_{M}\right)^{2 / 3}\left[\left(\Omega_{M}+\Omega_{V}-1\right)\left(\frac{2}{\Omega_{M}}\right)^{2 / 3}-\epsilon_{p}\right] \\
\times & {\left[\Omega_{M}^{4 / 7}-\Omega_{V}+\left(1+\frac{1}{2} \Omega_{M}\right)\left(1+\frac{1}{70} \Omega_{V}\right)\right]^{-1} . }
\end{aligned}
$$

A similar formula is possible if the field of fluctuations is normalised at any other epoch. Note that $\delta_{\lim } \propto\left(\epsilon_{p}+\right.$ constant $)$ and the time dependence of $\delta_{\lim }$ is given by that of $\epsilon_{p}$.

For the perturbation, the radius of maximum expansion can be calculated from Equation 1: this radius corresponds to the first positive root of the equation $2 G M+H_{0}^{2} \Omega_{V} a^{3}-$ $K a=0$ denoted by $a_{\max }$. This leads to a necessary and sufficient condition for the perturbation to collapse: that such a (finite) root exists. Because of the symmetry in Equation 1 , this model predicts that the perturbation will collapse to a singularity at a time equal to twice the time required to reach maximal expansion:

$H_{0} t_{\text {coll }}=2 \int_{0}^{a_{\max }^{*}}\left(\frac{2}{a^{*}}+\Omega_{V}\left(a^{*}\right)^{2}-\epsilon_{p}\right)^{-1 / 2} d a^{*}$

where we have changed from $a$ to $a^{*}=a H_{0}^{2 / 3} /(G M)^{1 / 3}$, and $a_{\max }^{*}$ is the first positive root of the equation:

$2+\Omega_{V}\left(a^{*}\right)^{3}-\epsilon_{p} a^{*}=0$.

Although collapse to a singularity does not occur in practice, the virialisation epoch is assumed to be similar to $t_{\text {coll }}$.

For perturbations that collapse, $\delta_{\lim }$ is called the 'critical' density and is denoted $\delta_{c}$. Equation then gives $\delta_{c}\left(\epsilon_{p}\right)$. Equations $8 \& 9$ give $t_{\text {coll }}\left(\epsilon_{p}\right)$, and the combination of these three Equations gives the required link between $\delta_{c}$ and the collapse time. Note that these Equations are independent of the perturbation mass, and therefore so is the link between the initial overdensity and the collapse time.

In practice we wish to use these Equations to calculate $\delta_{c}\left(z_{\text {coll }}, \Omega_{M}, \Omega_{V}\right)$ or $d \delta_{c}\left(z_{\text {coll }}, \Omega_{M}, \Omega_{V}\right) / d t$ where $z_{\text {coll }}$ is the collapse redshift. Unfortunately this is not easy as Equations $8 \& 9$ cannot be inverted to give $\epsilon_{p}\left(t_{\text {coll }}\right)$. The procedure adopted is as follows: the collapse time can be numerically determined from $z_{\text {coll }}$ using the Friedmann equation for the background cosmology. $\epsilon_{p}$ can be determined numerically using Equations \& \& and $\delta_{c}$ can be calculated using Equation $7 \delta_{c} / d t$ can be calculated numerically from $\delta_{c}\left(t_{\mathrm{coll}}\right)$ and is discussed further in the next Section.

For the subset of cosmological models with $\Omega_{V}=0$, the above procedure is simplified and analytic formula can be obtained for $\delta_{c}$. In this case, Equation 8 reduces to:

$H_{0} t_{\mathrm{coll}}=2 \int_{0}^{2 / \epsilon_{p}}\left(\frac{2}{a^{*}}-\epsilon_{p}\right)^{-1 / 2} d a^{*}$.

Making the substitution $\tan (\theta)=\left(2 / a^{*}-\epsilon_{p}\right)^{-1 / 2}$, this integral can be solved to give:

$H_{0} t_{\mathrm{coll}}=\frac{2 \pi}{\epsilon_{p}^{3 / 2}}$

We now show that these Equations provide the result of Gunn \& Gott 1972 for an Einstein-de Sitter cosmology. In this case, substituting Equation 11 into Equation 7 gives that:

$\delta_{c}\left(t_{\text {coll }}\right)=\frac{3}{20}\left(\frac{8 \pi}{H_{0} t_{\text {coll }}}\right)^{2 / 3}$.
We can now change from collapse time to collapse redshift to give:

$$
\begin{aligned}
\delta_{c}\left(z_{\mathrm{coll}}\right) & =\frac{3}{20}(12 \pi)^{2 / 3}\left(1+z_{\mathrm{coll}}\right) \\
& \simeq 1.69\left(1+z_{\mathrm{coll}}\right)
\end{aligned}
$$

which is the equation of Gunn \& Gott 1972.

\section{FROM A MASS FUNCTION TO A TIME DISTRIBUTION}

In this Section we show how to convert from a mass function to the distribution of times at which isolated halos of a given mass exist. First, we make the assumption that the mass of any clump is a monotonically increasing function of time so that the mass will increase between any two epochs. This is true for Press-Schechter theory (see paper I). Note that this mass growth is not constrained to be continuous and the mass is allowed to undergo instantaneous finite increases, or 'mass jumps'. Following this assumption, every epoch at which a halo exists should also be considered as a 'creation' epoch: every halo is a new isolated halo of some mass. The distribution of 'creation events' is therefore the same as the distribution of times at which the halos exist. Note that by definition this only applies to isolated halos which have not been subsumed into larger objects.

In this paper we have called this epoch the 'creation' time of a halo in order to avoid confusion with other authors definitions of the 'formation' time of a halo. Note that this semantic change was not adopted in paper I. The 'formation' time of a halo was defined by Lacey \& Cole (1993) as the latest time when the largest progenitor of a halo has a mass less than half that of the final halo. This definition makes sense if we are discussing a non-evolving quantity, say the existence of a galaxy halo, and wish to know when it was formed given that it exists at present day. However, suppose we do not know anything about the build-up of a halo before or after it has mass $M$ and only wish to know when it was likely to have existed. This Lacey \& Cole definition of 'formation' cannot help us for we do not know the time and mass from which to determine progenitors: progenitors of what?

In order to calculate the probability density function (pdf) of the times at which halos exist, we consider the set of all possible times and all possible halo masses. This is the 'sample space' of our 'experiment'. The experiment consists of choosing a particle, or small mass element, and an 'event' is given by any subset of the sample space: for instance that the particle is part of a halo of mass $M_{1}<M<M_{2}$ created at time $t_{1}<t<t_{2}$, or that the particle inhabits a halo of mass $M$, created at time $t$.

Denoting a generic pdf by the function $f$, the mass function is given by $f(M \mid t) d M$, the distribution of halo masses at a given epoch. This is equal to $M n(M) / \rho$ where $n(M)$ is the number density of halos. The pdf we wish to determine is given by $f(t \mid M) d t$, the distribution of times at which halos of mass $M$ were created. Note that our assumption of monotonic mass growth means that $t$ is the same variable in $f(M \mid t) d M$ and $f(t \mid M) d t$. These pdfs are then related by the following formula, based on Bayes' theorem: 
$f(t \mid M) d t=\frac{f(M \mid t) d M f(t) d t}{\int_{0}^{\infty}[f(M \mid t) d M f(t)] d t}$,

where $f(t) d t$ is the normalised prior for time, or the distribution of creation events in time given no information about the mass of halo. In paper I we calculated the prior using the Brownian random walks invoked in PS theory with a sharp $k$-space filter. In order not to bias the distribution of up-crossings within this model, we assumed a uniform prior for $\delta_{c}$.

The reason the prior is uniform in $\delta_{c}$ follows from the STHC model. Within this model, it is the density $\delta$ associated with a particle that is important, and the barrier has to move from $\delta$ to $\delta-d \delta$ for 'halo creation' to have occurred. Given that the mass of all clumps monotonically increases, all particles will be associated with creation events at any $\delta_{c}$. Following these two observations, any two equal width intervals in $\delta_{c}$ should contain equal 'numbers' of halo creation events.

The derivation presented in the previous Section showed that for the STHC model, the link between the critical overdensity and the collapse time is independent of the perturbation mass. Therefore, given no information about the mass contained within a perturbation, the pdf for the time at which the perturbation collapses should be assumed to be proportional to the time derivative of $\delta_{c}(t)$. This gives the rate at which the collapse threshold $\delta_{c}(t)$ crosses the initial overdensities. This can be calculated numerically from the following formula:

$$
\begin{aligned}
& \frac{d \delta_{c}}{d t} \propto \frac{d \epsilon_{p}}{d t}= \\
& {\left[\frac{d}{d \epsilon_{p}}\left(\int_{0}^{a_{\max }^{*}\left(\epsilon_{p}\right)}\left(\frac{2}{a^{*}}+\Omega_{V}\left(a^{*}\right)^{2}-\epsilon_{p}\right)^{-\frac{1}{2}} d a^{*}\right)\right]^{-1},}
\end{aligned}
$$

where $a_{\max }^{*}$ is the first positive root of the equation $2+$ $\Omega_{V}\left(a^{*}\right)^{3}-\epsilon_{p} a^{*}=0$. Note that for cosmologies with $\Omega_{V}=0$, the above equation can be analytically solved as for Equation 11, and the derivative $d \delta / d t$ is proportional to $t^{-5 / 3}$.

Unfortunately, $d \delta_{c}(t) / d t$ cannot be normalised so that it integrates over all time to give unity. This means that we cannot simply take a multiple of $d \delta_{c}(t) / d t$ as the prior for the collapse time. However, we can still use Equation 14 by making use of a mathematical trick and placing an arbitrary upper limit on $t, t_{u}$, which can be removed later without affecting the result. This gives that:

$f(t \mid M) d t=\lim _{t_{u} \rightarrow \infty}\left[\frac{f(M \mid t) d M f\left(t, t_{u}\right) d t}{\int_{0}^{t_{u}}\left[f(M \mid t) d M f\left(t, t_{u}\right)\right] d t}\right]$

The connection between the mass function and the creation rate of halos presented in this Section is consistent with that of paper I: the prior for time used is exactly the same. We have merely shown that adopting the STHC model for the rate at which structures are created allows any mass function to be converted to give the pdf of the time at which a halo of a given mass is created.

\section{THE RELATION WITH THE MULTIPLICITY FUNCTION}

Changing variables from mass to a function of

$\nu \equiv \frac{\delta_{c}}{\sigma_{M}}$

alters the form of the standard PS mass function to one which is invariant with respect to time. Here $\sigma_{M}$ is the rms fluctuation of the initial density field smoothed with a tophat filter on a scale related to mass $M$. Unless stated otherwise we change variables in the mass function from $M$ to $\ln \nu(M, t)$. The normalised pdf $f(\ln \nu \mid t)$ is called the multiplicity function and is related to the mass function by:

$f(M \mid t)=\left.A f(\ln \nu \mid t) \frac{\partial \ln \nu}{\partial M}\right|_{t}$,

where $A$ is a normalisation constant. Note that we have retained the condition on time in $f(\ln \nu \mid t)$, to emphasise that we are still concerned with the distribution of halos at a particular epoch. Although the multiplicity function has a form which is invariant with respect to time, it still gives the distribution of $\ln \nu$ we would expect for halos given a particular time. This is not the same as the the distribution of $\ln \nu$ we would obtain if we chose halos at random in both mass and time, or the distribution of $\ln \nu$ we would obtain if we chose halos only of a particular mass.

If the mass function can be written in a form which is independent of time as described above, then under the same change of variables, the creation rate becomes independent of halo mass. The resulting pdf $f(\ln \nu \mid M)$ is now only valid if we are examining the distribution of halos at fixed mass. Following the notation adopted above, this is given by:

$f(t \mid M)=\left.A f(\ln \nu \mid M) \frac{\partial \ln \nu}{\partial t}\right|_{M}$.

\section{THE JOINT DISTRIBUTION OF HALOS IN MASS AND TIME}

Because the mass of each halo is assumed to monotonically increase with time, within any interval of mass and time, an infinite number of 'creation events' occur. This means that the joint probability of the existence of a halo in both mass and time cannot be properly normalised.

Equation 16 gives the link between two pdfs, the mass function and the creation rate using a mathematical trick to cope with an un-normalised prior in time. The numerator of this equation is the joint distribution of halos in mass and time, $f(M, t) d M d t=f(M \mid t) d M f(t) d t$. The denominator is not a function of time: it only normalises the resulting formula so $f(t \mid M) d t$ integrates to unity. Following this argument, given a mass function, multiplying by $d \delta_{c}(t) / d t$ creates a function with both the correct mass and time behaviour. This joint distribution function (not a pdf) has the same mass dependence as $f(M \mid t)$ and the same time dependence as $f(t \mid M)$.

As an example we consider the fitting function of Sheth \& Tormen (1999) to the multiplicity function determined from the results of N-body simulations for different cosmological parameters:

$\frac{M n(M)}{\rho} d M=f(M \mid t) d M=f(\ln \nu \mid t) d \ln \nu$ 


$$
=A \sqrt{\frac{2}{\pi}}\left(1+\frac{1}{\nu^{\prime 2 p}}\right) \nu^{\prime} e^{-\nu^{\prime 2} / 2} d \ln \nu,
$$

where $\nu^{\prime}=a^{1 / 2} \nu$ and $a \& p$ are parameters. Note that Sheth $\&$ Tormen displayed this formula using a different notation to that adopted here, although parameters $a$ and $p$ are the same in both cases. $A$ is determined by requiring that the integral of $f(\ln \nu \mid t)$ over all $\ln \nu$ gives unity. Sheth \& Tormen found best fit parameters $a=0.707$ and $p=0.3$ for their simulations and group finding algorithm. The standard PS multiplicity function has $a=1, p=0$ and $A=1 / 2$. Unless stated otherwise, by standard PS theory, we refer to the adoption of this multiplicity function combined with top-hat filtering (to calculate $\sigma_{M}^{2}$ ). In order to convert this function to provide a model of both the time and mass of halo creation events, all we need to do is to multiply by $d \delta_{c} / d t$.

For standard PS theory, writing $\nu$ explicitly in terms of $\sigma_{M}^{2}$ and $\delta_{c}$ we find that the joint distribution of the existence of a halo in mass and time reduces to:

$$
\begin{aligned}
f(M, t) & d M d t=\frac{\delta_{c}}{(2 \pi)^{1 / 2} \sigma_{M}^{3}} \\
& \times \exp \left(-\frac{\delta_{c}^{2}}{2 \sigma_{M}^{2}}\right)\left|\frac{d \sigma_{M}^{2}}{d M}\right|\left|\frac{d \delta_{c}}{d t}\right| d M d t,
\end{aligned}
$$

Although not normalised, such a formula integrated over any two areas of the mass-time plane will provide the correct relative number densities.

Note that this is not the same formula as obtained by simply multiplying the mass function with the creation time distribution at fixed mass. This would be inconsistent within a Bayesian framework and would produce a joint density function which lacks the correct mass and time behaviour: the form of each conditional pdf is altered by the other. Care should therefore be taken when using the creation rate in models which also include the mass function.

\section{THE RELATION WITH MERGER EVENTS}

So far, we have only been concerned with the epoch at which a halo is created. However, there is an important distinction between major mergers and the slow accretion of mass when applying the results in models of certain cosmological phenomena. For instance, only violent merger events are thought to be important for starbursts and quasar activation. In paper I, we showed that for standard PS theory with a sharp $k$-space filter, if mass jumps in a particular trajectory correspond to merger events, then the distribution of mergers is the same as that of the build-up of matter from all types of creation event. This is because the trajectories are Brownian random walks which have the special property that their form is independent of the initial point.

Given only the mass function and the assumptions outlined above it is not possible to determine how each clump increases in mass, only the distribution of times at which it reaches a certain mass. More information about the buildup of individual clumps is required before the distribution of major mergers can be determined. Such information is available in PS theory and follows from the argument that each trajectory gives the history of the halo masses in which a particular small mass element resides.

\begin{tabular}{cccccc} 
model & $\Omega_{M}$ & $\Omega_{V}$ & $\Gamma$ & $\sigma_{8}$ & $\mathrm{~h}$ \\
\hline$\Gamma$ CDM & 1 & 0 & 0.25 & 0.64 & 0.5 \\
OCDM & 0.3 & 0 & 0.15 & 0.85 & 0.5 \\
$\Lambda$ CDM & 0.3 & 0.7 & 0.15 & 0.85 & 0.5
\end{tabular}

Table 1. Table showing the parameters of the different cosmological models adopted in the three N-body simulations.

\section{DESCRIPTION OF THE NUMERICAL SIMULATIONS}

A direct approach to modelling structure formation is to simulate the evolution of the mass density of the Universe using a distribution of softened particles. We have run three such simulations using the Hydra N-body, hydrodynamics code (Couchman, Thomas \& Pearce 1995) with $128^{3}$ dark matter particles to model the build-up of halos for three different cosmological models, described in Table 1 .

In order to determine the rate at which halos are created within these simulations, we output particle positions at a large number of times. For the $\Gamma$ CDM simulation, we output particle positions at 362 different epochs, separated by approximately equal intervals in time. For the OCDM simulation the number of outputs was 345 and for the $\Lambda$ CDM simulation, 499. The box size chosen was $100 \mathrm{~h}^{-1} \mathrm{Mpc}$ for all three simulations which gave a particle mass of $2.6 \times 10^{11} M_{\odot}$ for $\Gamma \mathrm{CDM}$ and $7.9 \times 10^{10} M_{\odot}$ for the other two simulations. Groups of particles were found for each output using a standard friends-of-friends algorithm with linking length set to $b=0.2$ times the mean interparticle separation.

\section{FITTING TO THE MASS FUNCTION}

The multiplicity function averaged over all output times is presented from each of the simulations in Fig. 1. Here we have only considered groups containing over 45 particles in order to limit the number of false detections due to numerical effects. In compiling the data in this way, we have assumed that converting from mass to $\ln \nu$ does indeed convert the form of the mass function into one which is independent of epoch. This Figure has been produced in such a way as to be directly comparable with figure 2 of Sheth \& Tormen (1999). For comparison we also plot their best fit model and the predictions of standard PS theory.

We have also plotted the model of Sheth \& Tormen (1999) (Equation 20) after allowing the parameters to vary to simultaneously fit the data from all three simulations. We find slightly different best fit parameters to those of Sheth $\&$ Tormen. Our best fit parameters are $a=0.774, p=0.274$, compared to standard PS theory $a=1, p=0$ and Sheth \& Tormen $a=0.707, p=0.3$. Note that the difference between our best fit values and those of Sheth \& Tormen could be explained by the different group finding algorithms used.

\section{COMPARISON BETWEEN THE ANALYTIC AND NUMERICAL HALO CREATION RATES}

Although we have argued that the monotonic increase in mass means that all epochs are 'creation' times for a given halo, we cannot simply compare the creation rate formulae 


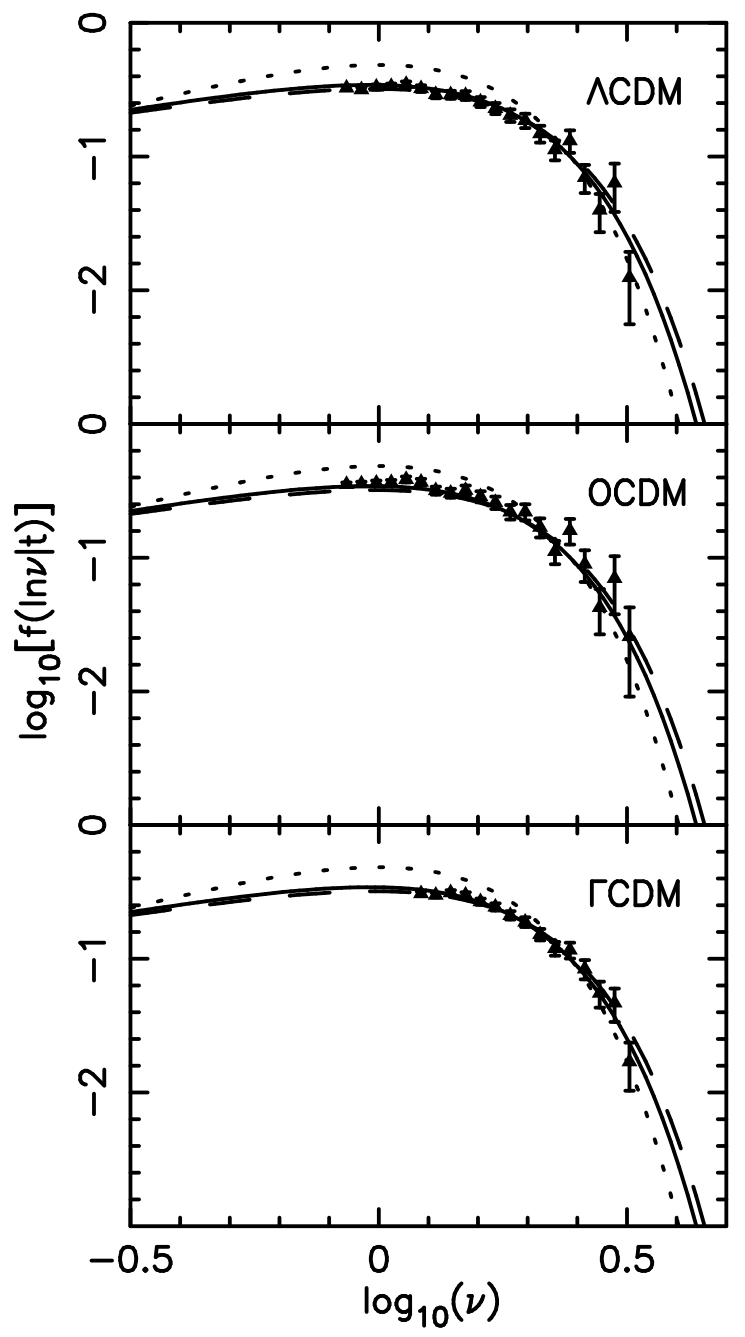

Figure 1. The measured multiplicity function from each of the three simulations analysed for all groups containing over 45 particles (solid triangles plotted with Poisson error bars). See Section 7 for details of these simulations. In compiling these data in this way we have assumed that the transfer of variables from $M$ to $\ln \nu$ where $\nu \equiv \delta_{c} / \sigma_{M}$ does make the distribution of masses independent of epoch as found by Sheth \& Tormen (1999). For comparison we have also plotted the best fit model to their data (dashed line) and the predictions of standard PS theory (dotted line). The solid line shows the best fit model to our data allowing the parameters of the Sheth \& Tormen (1999) fitting function to vary.

with the distribution of halo numbers at different epochs: each halo should only be counted once. To determine the distribution of creation times of halos of mass $M$, we therefore sequentially analysed the FOF output from $z=50$ to present day. All halos of mass $>M$ were examined at each epoch to determine whether they were 'new'. The definition of 'new' adopted was that at least half of the particles in a halo were not included in any halo of mass $>M$ at a previous output time. The number of these halos in the required mass range was taken to be the minimum number which could have been created between that output time and the previous one. In order not to miss creation events where a halo was created and subsumed into a larger halo all within the time interval between two outputs, we anal- ysed the progenitors of all new halos with mass greater than the required range. Those with a progenitor distribution at the previous step which could sum to a halo of the required mass were recorded as a possible halo of the required mass. In this way we determined the minimum and maximum mass which could have been created in each time interval between output from the simulation.

In Fig. 2 we plot the creation rate for halos within two narrow mass ranges. In order to obtain the maximum number of creation events, we have used relative low numbers of particles in each group. Data are plotted for groups of between $45-50$ and $100-110$ particles. These distributions are compared with the three multiplicity functions plotted in Fig. 2, converted into creation rates by multiplying by $d \delta / d t$ for halos of mass equivalent to 45 or 100 particles. These curves have been normalised to the low redshift data.

All of the models reproduce the decrease in creation events to present day seen in the simulations. As output from the simulation occured after approximately equal intervals of time, the high redshift data suffers as the intervals contain relatively more creation events. This means that we cannot precisely follow the build-up of the clumps, and the difference between the maximum and minimum mass which could have been created in each bin is increased. This is particularly noticable in the OCDM simulation where halos are created at earlier times and we have fewer outputs from the simulation.

However, there is evidence that the solid line (calculated from the best fit to the mass function) also fits the creation rate data the best out of the three models plotted. As a rough guide to this, the root mean square value between the plotted data points and the model is 3.4 for this curve, compared to 6.7 for standard PS theory, and 5.0 for the best fit model of Sheth \& Tormen (1999). Note that the form of the creation rate is strongly dependent on the parameter $a$ in Equation 20, and only weakly dependent on parameter $p$. This is consistent with the importance of these parameters for the mass function: parameter $a$ controls the position of the high-mass cut-off, whereas parameter $p$ controls the lowmass tail of the distribution.

\section{CONCLUSIONS}

We have demonstrated a simple method for linking any mass function to the corresponding distribution of times at which isolated halos of a given mass are created. In order to provide this link we adopted the assumption that the time scales of interest are those over which the mass of every clump can be thought of as monotonically increasing. The prior for the collapse time was estimated using the STHC model which ties in directly with PS theory, although the method does not use any of PS theory beyond that of the STHC model. We have presented a new derivation of the link between the collapse time and initial overdensity for this model which explicitly shows that this link is independent of the halo mass and is applicable in any Friedmann cosmology. Multiplying the mass function by a function with no mass dependence and proportional to the time derivative of the critical overdensity then provides a joint density function with the correct behaviour for the creation of a halo in mass and time. Integrating over the resulting joint density function will give 


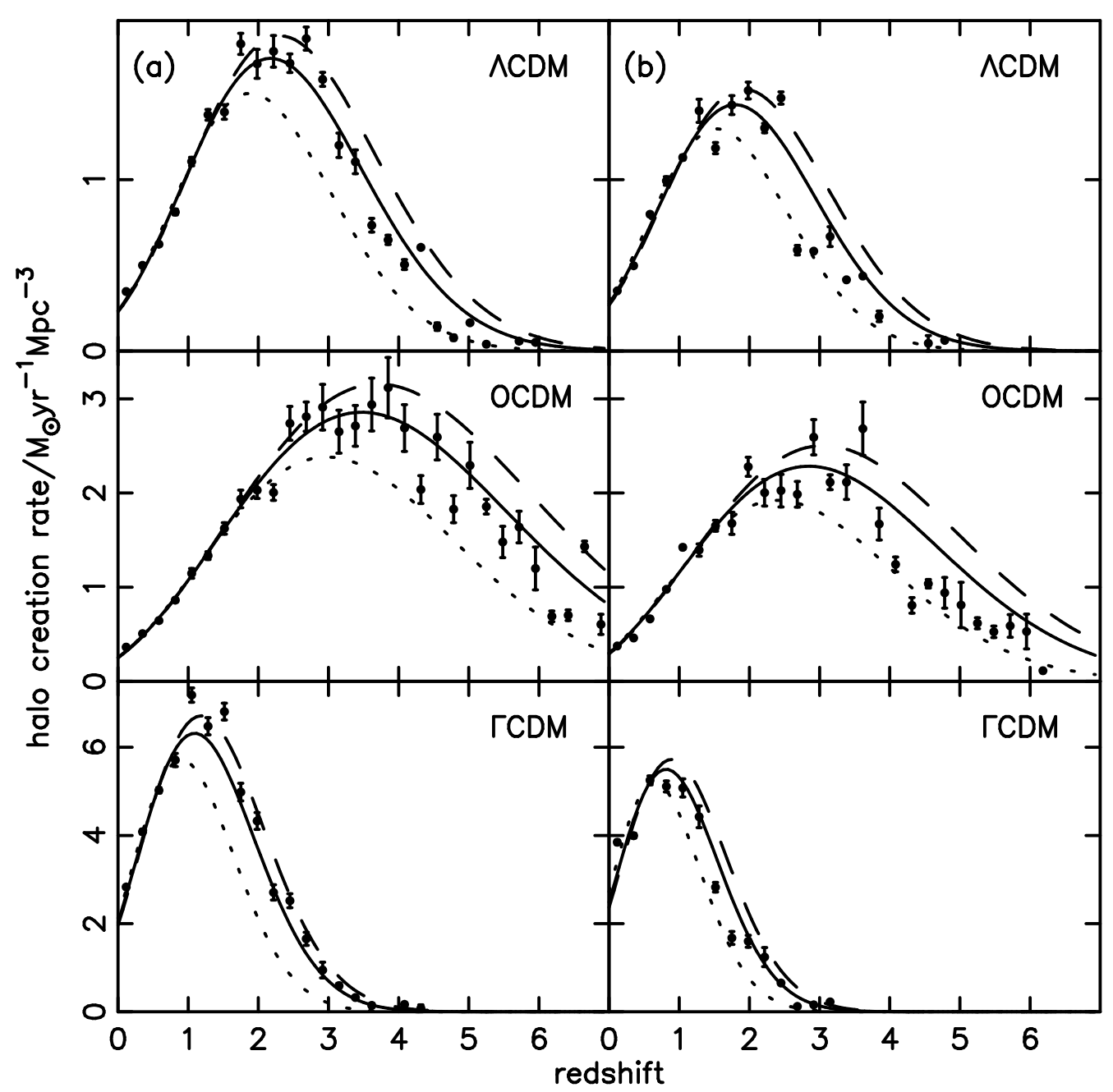

Figure 2. The creation rate of halos as determined from the three cosmological simulations (solid circles) for a) 45-50 particles in a halo and b) 100-110 particles. These group sizes correspond to masses of aproximately a) $1.2 \times 10^{13} M_{\odot}$ for $\Gamma$ CDM and $3.6 \times 10^{12} M_{\odot}$ for the two other cosmologies, and b) $2.6 \times 10^{13} M_{\odot}$ for $\Gamma \mathrm{CDM}$ and $7.9 \times 10^{12} M_{\odot}$ for the two other cosmologies. The error bars show the binned minimum and maximum mass which could have been created in halos of the required masses in each time interval. Symbols are plotted half way between the two. Note that the errors bars do not include counting errors. For comparison we also plot the three models of the mass function multiplied by $d \delta_{c} / d t$ with lines as in Fig. 1. The models have been normalised to the low redshift data.

the correct relative number densities of halos within different mass and time intervals.

We have extended the analysis of N-body simulation results presented in paper I to cover three simulations of the build-up of dark matter within different cosmological models. Rather than using PS theory, we have demonstrated how a fit to the mass function may be converted to give a creation rate. Out of the three functions we have compared to the hass function data. the best fit model for these data when converted to a creation rate also fits the creation rate data the best. This gives us confidence that the formalism presented here is sound, and should give accurate results in more gфneral situations, in particular non-Gaussian models.

\section{ACKNOWLEDGEMENTS}

We are grateful for the use of the Hydra N-body code (Couchman, Thomas \& Pearce 1995) kindly provided by the Hydra consortium.

\section{REFERENCES}

Bond J.R., Cole S., Efstathiou G., Kaiser N., 1991, ApJ, 379, 440 Carroll S.M., Press W.H., Turner E.L., 1992, ARA\&A, 30, 499

Couchman H. M. P., Thomas P.A., Pearce F.R., 1995, ApJ, 452, 797

Eke V.R., Cole S., Frenk C.S., 1996, MNRAS, 282, 263

Granato G.L., Silva L., Monaco P., Panuzzo P., Salucci P. De Zotti G., Danese L., 1999, MNRAS submitted, astro$\mathrm{ph} / 9911304$

Gunn J.E., Gott J.R., 1972, ApJ, 176, 1

Kitayama T., Suto Y., 1996, ApJ, 469, 480

Lacey C., Cole S., 1993, MNRAS, 262, 627

Matarrese S.. Verde L., Jimenez R., 2000, ApJ submitted, astro$\mathrm{ph} / 0001366$

Peacock J. A., Heavens A. F., 1990, MNRAS, 243, 133

Percival W.J., Miller L., 1999, MNRAS, 309, 823

Press W., Schechter P., 1974, ApJ, 187, 425

Sheth R.K., Tormen G., 1999, MNRAS, 108, 119

Somerville R.S., Primack J.R., 1999, MNRAS accepted, astro$\mathrm{ph} / 9802268$ 